A N N A L E S Annales de Bretagne et des Pays de l'Ouest

Anjou. Maine. Poitou-Charente. Touraine

109-4 | 2002

Les étrangers dans l'Ouest de la France (XVIII $-{ }^{\mathrm{e}} \mathrm{XX} \mathrm{C}^{\mathrm{e}}$ siècle)

\title{
Les réfugiés politiques en Bretagne (1830-1848)
}

\section{Patrick Harismendy}

\section{(2) OpenEdition}

1 Journals

Édition électronique

URL : http://journals.openedition.org/abpo/1509

DOI : 10.4000/abpo.1509

ISBN : 978-2-7535-1488-1

ISSN : 2108-6443

Éditeur

Presses universitaires de Rennes

Édition imprimée

Date de publication : 20 décembre 2002

Pagination : 41-58

ISBN : 978-2-86847-794-1

ISSN : 0399-0826

Référence électronique

Patrick Harismendy, "Les réfugiés politiques en Bretagne (1830-1848) 》, Annales de Bretagne et des

Pays de l'Ouest [En ligne], 109-4 | 2002, mis en ligne le 20 décembre 2004, consulté le 01 mai 2019.

URL : http://journals.openedition.org/abpo/1509; DOI : 10.4000/abpo.1509 


\title{
Les réfugiés politiques en Bretagne (1830-1848)
}

\author{
Patrick HARISMENDY \\ Professeur d'histoire contemporaine, \\ CRHISCO - Université Rennes 2
}

Par sa position excentrée, la Bretagne n'a pas constitué un pôle privilégié d'accueil de réfugiés politiques au XIX ${ }^{\mathrm{e}}$ siècle - pour l'essentiel sous la Restauration et la Monarchie de Juillet (moins de 0,12 \% de la population est d'origine étrangère en 1851 pour les quatre départements de la Bretagne actuelle). En même temps, les volumes restreints d'individus concernés en Ille-et-Vilaine, Côtes-du-Nord et Morbihan, pris ici comme espaces-tests, devraient permettre de suivre avec précision les flux ${ }^{1}$. Or, la réalité s'avère plus complexe. La modernité des procédures administratives d'accueil, d'enregistrement, d'affectation et de subsides est en effet démentie par les réalités locales et, surtout, des populations impossibles à réduire en un type unique. En effet, des cohortes identifiées voisinent avec des agrégats satellites, des constellations d'individus dissimulent de simples comètes, les uns passent, les autres s'arrêtent, s'intègrent ou meurent. On ne saurait donc surévaluer un phénomène qui, au fond, s'apparente à d'autres migrations, diasporas et refuges, qu'il s'agisse de travail, de coercitions religieuses ou d'une recherche de différentiel à la pauvreté. Dans l'absolu, l'examen des corpus nominatifs fournis par les sources administratives relève de plusieurs catégories dont il faudrait partir : ethno-politique pour l'identification des réfugiés; socio-professionnelle pour repérer les voies d'exil; culturelle dans la double perspective de l'accueil et de l'intégration. Mais on peut aussi s'interroger sur les procédures administratives elles-mêmes pour faire la part entre la norme et la relative inefficacité

1. Pour Ille-et-Vilaine et Morbihan, nous citerons - après vérifications - les dossiers à partir des mémoires de maîtrise que nous avons dirigés : DESIRABEL, Lucile, Les réfugiés politiques en France durant la première moitié du XIX siècle (Italiens, Portugais et Polonais), 1999, 103 p.; LE DEUFF, Olivier, Exilés et comploteurs - Les réfugiés politiques italiens, polonais et portugais en Ille-et-Vilaine durant la première moitié du XIXe siècle, 1999, 67 p.; MAHE, Fabienne, Les réfugiés politiques dans le Morbihan au XIX siècle (Polonais, Italiens et Espagnols), 134 p.; pour les Côtes-du-Nord, dépouillements personnels. À signaler aussi les articles pionniers de CORBES, Hippolyte, notamment, « Les émigrés polonais dans les Côtes-du-Nord de 1833 à 1870 ", Société d'émulation des Côtes-du-Nord, 1970, p. 80-97. 
d'un dispositif à grosses mailles. De sorte que le phénomène de refuge en dit peut-être plus sur l'État de tolérance - ou d'indifférence - que sur les exilés eux-mêmes avec le mérite, néanmoins, de pouvoir fournir des estimations, impossibles pour une Grande-Bretagne pratiquant la libre circulation des hommes, même pour cause politique ${ }^{2}$.

\section{Exils des peuples, exil des hommes}

Pour la période considérée, quatre ensembles nationaux ont fourni des exilés en Bretagne : la Pologne, le Portugal, l'Espagne et les Italies. Dans le principe, les individus sont identifiés à partir de la circulaire ministérielle du 24 septembre 1823 invitant les préfets à recenser les réfugiés présents dans leur département. Dans les faits, il faut attendre la loi du 24 avril 1832 pour y voir clair ${ }^{3}$. Cette loi suit un débat parlementaire houleux entre le Mouvement (sollicitant que la Grande Nation accueille des " compatriotes ") et la Résistance (affirmant, dans une logique censitaire, que l'absence de contribution financière ou matérielle des exilés ne peut en faire des Français). Naturellement, cette dernière interprétation prévalut et on le voit bien dans le cas d'Adalbert Lipinsky, de Joseph Jankowski et de Stanilas Bagienski qui ne bénéficient d'aucun traitement de faveur alors qu'ils avaient servi la France dans le passé ${ }^{4}$ : le premier, capitaine d'infanterie engagé dans la campagne de Prusse en 1806, blessé en 1809, fait chevalier de la Légion d'Honneur par l'Empereur en personne, blessé et fait prisonnier durant la campagne de Russie; le second, sous-officier d'artillerie depuis 1807, lui aussi décoré de la Légion d'Honneur, par Ney, en Russie; le dernier, capitaine d'artillerie sorti du rang, avait à son actif les campagnes de Russie, Allemagne, France (1814) et était aussi légionnaire depuis octobre 1813.

Le texte d'avril 1832 introduit néanmoins deux éléments capitaux : l'assignation à résidence et le versement de "subsides " en numéraires destinés, tout à la fois, à prévenir la mendicité et à permettre le contrôle individuel puisque le réfugié doit se présenter physiquement aux autorités municipales tous les quinze jours. Temporaires, ces allocations devaient préfigurer une intégration professionnelle dans la société française ${ }^{5}$. Tout déplacement, conditionné par l'obtention d'un passeport (souvent d'indigent) délivré, dans les termes du règlement de 1816, par un maire (jusque 1839) ou le corps préfectoral - à la condition expresse d'être désarmé, -

2. Lecture moins pessimiste que celle proposée par NoIRIEL, Gérard, "Représentations nationales et catégories sociales; l'exemple des réfugiés politiques ", DEvoTo, Fernando et GonCALVES-BERnALDO, Pilar, Émigration politique, une approche comparative - Italiens et Espagnols en Argentine et en France. XIX ${ }^{e}-X X^{e}$ siècles, Paris, L'Harmattan, 2001, p. 45-75.

3. Mondonico, Cécile, L'asile sous la Monarchie de Juillet. Les réfugiés étrangers en France de 1830 à 1848, Paris, thèse EHESS, dir. Gérard Noiriel, 1991.

4. Arch. dép. des Côtes-d'Armor, 4 M 198, 200, 202 (dossiers personnels).

5. Arch. dép. du Morbihan, 4 M 387, rappel du préfet aux sous-préfets (2 mars 1852); NoIRIEL, Gérard, Réfugiés et sans-papiers. La République face au droit d'asile XIXe-XXe siècles, Paris, Calmann-Lévy, 1991, p. 49. 
est donc suivi grâce au versement des subsides ${ }^{6}$. Ces derniers se distribuent en cinq " classes ", correspondant à la hiérarchie militaire (ou de la fonction publique), mais négligent les professions commerciales, industrielles, voire intellectuelles, ce qui pose parfois des problèmes de rattachement à telle ou telle classe.

Dans les trois départements examinés ici, les affectations et regroupements obéirent à trois modèles (comme un peu partout ailleurs, du reste). Le recours aux dépôts qui était préconisé avant la loi d'avril 1832, fut employé dans le Morbihan à Auray, Lorient, Vannes et Belle-Île, en Ille-etVilaine, à Rennes, Fougères, Hédé et Dol, enfin à Dinan dans les Côtes-duNord. À titre d'exemple, le très important dépôt de Fougères accueillit 42 officiers et 237 soldats portugais en septembre 1829, celui de Rennes, 209 personnes en janvier 1831. Or, bien que l'art. $1^{\text {er }}$ de la loi de 1832 en ait prononcé la dissolution, celle-ci ne devint effective qu'assez tard (17 mai 1833 pour Belle-Ile ${ }^{7}$ ). Néanmoins, la terminologie administrative conserva longtemps l'idée de " dépôt " - correspondant, en fait, aux assignations à résidence - puisqu'en 1834 et 1835, Auray et Lorient ressortaient à cette catégorie $^{8}$. L'assignation collective à résidence constitue donc le second type de traitement. La dissolution des grands dépôts de Moulins et Rodez (pour les Italiens), Bourges et Besançon (pour les Polonais), entre juillet 1832 et juin 1833, enfin de Tulle, Guéret et Châteauroux (pour les Espagnols, en mars 1834), qui furent le théâtre de rivalités et d'affrontements entre nationaux, détermina un afflux important de réfugiés en Bretagne puisque le tiers des consignés de Rodez, les deux tiers de Moulins et la quasi-totalité des bisontins y furent affectés ${ }^{9}$. Le double impératif de dissémination et de surveillance incita donc les autorités à choisir des communes de taille moyenne (le plus souvent des chefs-lieux d'arrondissement) et dotées de maires à poigne. Dans les Côtes-du-Nord, si l'on trouve des réfugiés à Lannion, Guingamp et Dinan, on constate que Moncontour est souvent préféré à Lamballe, de même Quintin à Loudéac. Quant aux individus isolés, formant la troisième catégorie de réfugiés, moins suspects de débordements collectifs, leur affectation s'effectue plus aisément au chef-lieu de département.

Évaluer le nombre de réfugiés constitue, de ce fait, une opération délicate que traduisent, d'ailleurs, certaines approximations au plan national (20000 selon Pierre Lequin pour les années 1830-1850, 30000 en 1840 et 14000 en 1845 pour Cécile Mondonico ${ }^{10}$ ). Il faut, en effet, distinguer les

6. NOIRIEL, Gérard, "Surveiller les déplacements et identifier les personnes. Contribution à l'histoire du passeport en France de la première à la troisième République ", Genèses, 30, mars 1998, p. 77-100.

7. Arch. dép. du Morbihan, 4 M 387.

8. Arch. dép. du Morbihan, 4 M 387 et 388.

9. Arch. dép. du Morbihan, 4 M 388, lettre du Préfet du Morbihan au maire d'Auray (27 juin 1833); Arch. dép. des Côtes-d'Armor, 4 M 204.

10. LEQUIN, Yves (dir.), La mosaïque France - Histoire des étrangers et l'immigration en France, Paris, Larousse, 1998, p. 326; Mondonico, Cécile, op. cit., p 88-89. 
affectations réelles (donc mises en œuvre), des mouvements périodiques (pour affaires de famille, agrément, causes médicales, voire attirance pour les bains de mer!), sans oublier les départs effectifs pas toujours faciles à cerner. De même que les dossiers départementaux ne donnent que des indications fugitives, très fragmentaires et souvent trompeuses, sauf à envisager la constitution, un jour, d'une base de données générale. Et encore faudrait-il s'en défier. Ainsi, parmi les deux polonais des Côtes-du-Nord émigrés aux États-Unis (contre 32 pour le Morbihan), l'un, le Dr Joseph Schuliger (parti en août 1833), revient à Saint-Brieuc, en mars $1838^{11}$. De même, Joseph Nesterowicz qui se vit interdire le mariage avec une indigente de Tréguier, contracte des dettes, est arrêté pour vol par la gendarmerie de Pontrieux, s'évade et fuit à Jersey, en 1834, mais se trouve réadmis aux subsides après avoir perdu son emploi de magasinier au port de Lorient, en août $1851^{12}$.

En termes de stocks, comme le propose Fabienne Mahé, 332 réfugiés politiques ont donc séjourné dans le Morbihan au XIX ${ }^{\mathrm{e}}$ siècle (143 Polonais, 117 Italiens et 72 Espagnols sachant, d'après une circulaire ministérielle du 11 avril 1841, que 8000 carlistes auraient résidé en France à cette date) ${ }^{13}$. Une analyse des flux traduit une autre réalité, plus proche des circulaires ministérielles du 6 mai et du 22 juillet 1833, bornant à 70 le nombre de réfugiés politiques admissibles dans les départements (hors dépôts), dont 15 à 20 au chef-lieu ${ }^{14}$ : en 1833, il y eut, en effet, jusqu'à 72 Polonais dans les Côtes-du-Nord et 71 dans le Morbihan. Pour les Côtes-du-Nord, le tableau ci-dessous - ne présentant ici que des valeurs médianes arrondies extraites des états mensuels de présences, entrées et sorties dans les arrondissements ${ }^{15}$ - montre que la proportion de réfugiés présents est grossièrement équivalente à celle des travailleurs étrangers (Suisses, Allemands et Belges); mais elle s'avère infime comparée aux Anglais (et à quelques Américains anecdotiques) présents au long de l'année ou à la belle saison, surtout dans l'arrondissement de Dinan ${ }^{16}$.

Du reste, et même si l'on ne dispose que d'un chiffre isolé, les déplacements de réfugiés ne sauraient se comparer aux 966 voyageurs ayant traversé les Côtes-du-Nord en $1826^{17}$. Cependant, les comptes mensuels permettent de mieux cerner les mouvements immédiats. On peut notamment suivre les 94 réfugiés antérieurement au dépôt de Dol $^{18}$.

11. Arch. dép. des Côtes-d'Armor, 4 M 203.

12. Arch. dép. des Côtes-d'Armor, 4 M 202; Arch. dép. du Morbihan, 4 M 357.

13. MAHE, Fabienne, op. cit., p. 9-12; Arch. dép. des Côtes-d'Armor, 4 M 205.

14. Arch. dép. des Côtes-d'Armor, 4 M 199.

15. Arch. dép. des Côtes-d'Armor, 4 M 108-110.

16. Sur ce proto-tourisme, CORBES, Hippolyte, «L'immigration anglaise dans les Côtesdu-Nord ", Société d'émulation des Côtes-du-Nord, 1962, p. 118.130.

17. Arch. dép. des Côtes-d'Armor, 4 M 250 : registre bimestriel d'identification des voyageurs traversant le département en 1826.

18. Arch. dép. d'Ille-et-Vilaine, 4 M 359. 
Tableau 1 - Recensement des étrangers présents dans les Côtes-du-Nord (1832-1845)

\begin{tabular}{|l|c|c|c|}
\hline & Personnes en villégiature & Réfugiés politiques & Travailleurs étrangers \\
\hline 1832 & 212 & 8 & 21 \\
1833 & lacunes & lacunes & lacunes \\
1834 & 238 & 52 & 27 \\
1835 & lacunes & lacunes & lacunes \\
1836 & 324 & 33 & 30 \\
1837 & 294 & 29 & 28 \\
1838 & 287 & 26 & 27 \\
1839 & 308 & 23 & 22 \\
1840 & 313 & 26 & 35 \\
1841 & 357 & 26 & 40 \\
1842 & 410 & 30 & 40 \\
1843 & 456 & 31 & 45 \\
1844 & 439 & 18 & 41 \\
1845 & 337 & 15 & 42 \\
\hline
\end{tabular}

Tableau 2 - Réfugiés portugais restant en France

\begin{tabular}{|l|c|}
\hline Restent à Dol & 35 \\
Vont à Fougères & 4 \\
Vont à St-Malo et Brest & 2 \\
Vont à Rennes & 21 \\
Vont dans les Côtes-du-Nord & 12 \\
Vont en Mayenne & 6 \\
Vont en Loire-Inférieure & 2 \\
Vont à Paris & 2 \\
\hline
\end{tabular}

On connaît, de même, le destin des 72 Polonais affectés dans les Côtesdu-Nord, en 1833, - dont 47 venus de Besançon - jusqu'à la fin de 1834 à partir des registres d'enregistrement nominatif et de versement des subsides ${ }^{19}$.

\section{Tableau 3 - Réfugiés polonais restant en France}

Restent dans les Côtes-du-Nord

Vont dans d'autres départements bretons

Vont en Normandie

Vont à Paris

Vont à Bordeaux

Vont dans d'autres régions françaises
Tableau 4 - Réfugiés polonais quittant la France

\begin{tabular}{|l|l|}
\hline Partent en Angleterre & 6 \\
Partent en Belgique & 7 \\
Partent au Portugal & 1 \\
Partent aux États-Unis & 2 \\
Sont expulsés & 2 \\
\hline
\end{tabular}

19. Arch. dép. des Côtes-d'Armor, 4 M 198-199. 
La fiabilité de ces indications est pourtant relative, car certains départs pour Saint-Malo, la Normandie, voire la Belgique, préfigurent des passages pour l'Angleterre. Au bout du compte, l'analyse comptable n'est pertinente qu'en raisonnant en termes de cycles (donc de stabilité) des populations réfugiées. Les seuils d'étiage seraient donc, et toujours dans les Côtes-duNord, pour les Portugais entre novembre 1829 et janvier 1832 (10 à 15 personnes $)^{20}$, pour les Polonais, la période allant d'octobre 1833 à juin 1844 (25 à 17 résidents avant une brusque stabilisation à 8); pour les Italiens de juillet 1834 à juillet 1838 (5 à 3), pour les Espagnols (arrivés en avril 1841), de décembre 1841 à avril 1844 (de 9, puis 13 à 8). La chronologie est voisine dans le Morbihan, comme en témoigne le tableau suivant ne prenant pourtant en compte que les arrivées :

Tableau 5 - Cycle de présence des réfugiés (admis aux subsides) dans le Morbihan (1830-1859)

\begin{tabular}{|l|c|c|c|c|c|c|}
\hline & $1830-1834$ & $1835-1839$ & $1840-1844$ & $1845-1849$ & $1850-1854$ & $1855-1859$ \\
\hline Espagnols & 0 & 0 & 31 & 15 & 19 & 4 \\
Polonais & 85 & 4 & 2 & 3 & 5 & 2 \\
Italiens & 65 & 9 & 18 & 0 & 2 & 0 \\
Total & 150 & 13 & 51 & 18 & 26 & 6 \\
\hline
\end{tabular}

Autrement dit, deux grands profils se dessinent : d'une part, des contingents de réfugiés plutôt stables sur de longues périodes; d'autre part, des individus mobiles, soit pour motifs personnels, soit, le plus souvent, affectés au gré de l'Administration.

Subsiste, enfin, un dernier problème. À savoir que le contrôle de tous les réfugiés - rappelé notamment par la circulaire du 15 février 1838 - n'est plus effectif au début du Second Empire qui ne retient désormais que les subventionnés dans ses statistiques. Par conséquent, et en quelque sorte, l'Administration génère elle-même les voies d'une intégration formelle, bien que celle-ci reste assez éloignée des réalités factuelles.

\section{Les territoires de l'exil}

Pour l'essentiel, le principe de surveillance s'applique dans trois domaines (politique, spatial et comportemental) avec, dans l'idéal, cette idée formulée par le rapporteur Bresson, le 11 avril 1833, que « les étrangers se mêlent tellement à notre population que le gouvernement seul sache où sont les réfugiés".

Dans le domaine politique, les craintes de l'Administration concernent autant les éventuelles agitations républicaines ou légitimistes que les provocations supposées à l'égard des Puissances, au risque de renforcer certains stéréotypes sur l'" étranger ». L'exil est donc subordonné à une dou-

20. Arch. dép. des Côtes-d'Armor, 4 M 204. 
ble privation : celle de l'activité politique et celle de la nationalité, le réfugié se devant d'être apolitique et apatride (faute de naturalisations avant 1849) ${ }^{21}$. Les Portugais, généralement considérés comme exemplaires, d'autant qu'ils restent soumis à leurs officiers - chargés de négocier avec les autorités si besoin est - dans les dépôts, inquiètent peu. Sans doute est-ce pour cela qu'en dépit des craintes du sous-préfet de Saint-Malo concernant le maintien du dépôt de Dol, " petite ville où l'esprit révolutionnaire est exalté " et qui " renferme un très grand nombre de petits propriétaires oisifs " que la présence d'étrangers " pourrait rendre dangereux ${ }^{22}$ ", celuici soit demeuré actif jusqu'en 1832. Mais on se méfie d'éventuels contacts avec les libéraux fougerains. En témoigne, notamment, la rectification de la pierre tombale du sergent Joseph-Joachim Macedo, mort le 21 juin 1829 à 30 ans, " ami de sa patrie ", qui " avait pris les armes contre le tyran qui l'agressait " et qui avait abandonné les " champs que le Tage et le Douro baignent " car " la cause du crime " avait triomphé. On imposa donc la formule plus prudente : "Éloigné de sa patrie par les troubles qui l'agitent, il vint chercher un asile en France, et finit ses jours sur cette terre hospitalière $^{23}$. " Également nombreux et très surveillés, les réfugiés polonais causent peu de troubles politiques malgré la crainte de les voir s'affilier à une société secrète (en fait la Société démocratique polonaise qui ne compte qu'un cadre en Bretagne, Ignace Pluranski, dans le Finistère ${ }^{24}$ ).

En revanche, Espagnols et Italiens posent problème. Les premiers, qui comptent une majorité d'officiers supérieurs (11 lieutenants-colonels sur les 15 Espagnols résidants dans les Côtes-du-Nord en août 1843), sont aidés par les légitimistes, tel Estan Arastay dont la pension dans une auberge de Mûr-de-Bretagne est payée, en 1843, par la famille Keranflec'h de Kernezné (de Saint-Gilles-Vieux-Marché), un autre s'abritant (en 1846) chez le vicomte de Saisy de Kerampuil (à Glomel); il s'agit là de deux familles d'officiers légitimistes, démissionnaires en 1830, et donnant des députés de cette sensibilité sous la III ${ }^{\mathrm{e}}$ République. On peut y ajouter la famille d'Abbyville, soutien avéré du fils de Don Carlos dans le château du Chatellier (près de Dinan). Plus paranoïaque, sans doute, est ce rapport du capitaine de gendarmerie de Saint-Brieuc (10 août 1843), affirmant que deux Espagnols passent régulièrement la frontière entre Finistère et Côtes-du-Nord, et " sollicitent des secours particuliers dans les châteaux et chez les ecclésiastiques " entre Paimpol et Merdrignac avec des lettres signées Cadoudal $^{25}$ ! La conjonction de ces bruits détermine en tout cas la décision

21. Réalité voisine soulignée par MoReluI, Anne, « Belgique, terre d'accueil? Rejet et accueil des exilés politiques en Belgique de 1830 à nos jours ", L'émigration politique en Europe aux XIX et XXe siècles, Rome, Collection de l'École française de Rome, 146, 1991, p. $117-128$.

22. Arch. dép. d'Ille-et-Vilaine, 4 M 359 : lettre du sous-préfet de Saint-Malo du 21 décembre 1829 .

23. Arch. dép. d'Ille-et-Vilaine, 4 M 357.

24. Arch. dép. des Côtes-d'Armor, 4 M 199; Arch. dép. d'llle-et-Vilaine, 4 M 353.

25. Arch. dép. des Côtes-d'Armor, 4 M 205. 
ministérielle du 13 décembre 1843 étendant à tout l'Ouest (Côtes-du-Nord, Morbihan et Finistère compris) les interdictions de résidence définies, le 12 juillet 1839, puis le 4 juillet 1841, pour les Espagnols. L'application de cette mesure de sécurité publique aux 13 Espagnols présents en novembre 1843 est cependant limitée, puisqu'en janvier-mars 1844, ils sont encore 5 à résider (dont le colonel Arnoz, resté à Lamballe, et trois individus nonsubventionnés demeurés à Dinan) ${ }^{26}$. Plus singulier est le destin du lieutenant-colonel Erlanz, employé chez un notaire de Guingamp (M. de Floyd) qui part effectivement, en janvier 1844, mais revient en août, y reste jusqu'en 1847 pour aller à Agen (on ne sait pourquoi), négocie encore un retour à Guingamp pour y travailler chez son protecteur, en mai 1849, lorsqu'il apprend son amnistie, le 8 juin 1849, et rentre finalement en Espagne par Bayonne ${ }^{27}$. De fait, l'interdiction collective de séjour, répétée le 9 décembre 1849, explique encore qu'en 1853-54, 6 colporteurs ou représentants de commerce d'origine carliste doivent obtenir de l'Administration des permis de circuler dans les Côtes-du-Nord ${ }^{28}$.

Quant aux Italiens, diverses préventions s'attachent à eux. Plus volontiers suspects d'usurpation dans le statut de réfugié politique (comme Louis Ceppari, toscan, qui s'avère n'être qu'un émigré volontaire ${ }^{29}$, tout comme Bernabo-Silotara, Brucalassy, Montolfi, Pantassi ou Generoso Serra professeur d'italien non réfugié ${ }^{30}$ ), ou d'attribution indue de subsides (comme Jacques Tournon, fils d'un officier français ayant servi de juin 1793 à septembre 1814 et mort à Modène en $1820^{31}$ ), la rareté des militaires dans leurs rangs en fait des conspirateurs tout désignés, tels le toscan Louis Pozzi expulsé vers l'Angleterre en $1834^{32}$. D'autres figures sont plus impressionnantes, car si le piémontais François Germani est seulement signalé (en juin 1839) au cas où il viendrait d'Angleterre pour comploter, le piémontais Antoine Ferranti (de son vrai nom Antonio Ferranni) a la stature d'un personnage de roman, comme en témoigne la minutieuse reconstitution opérée par Olivier Le Deuff et qu'on pourrait encore compléter dans d'autres départements sans doute:

- mars 1834 : signalé à Avignon

- 25 mars 1834 : arrêté d'expulsion pour participation à l'expédition de Savoie

- 12 juillet 1834 : arrivée à Calais, pour passage en Angleterre

- 26 juillet 1834 : signalé de nouveau à Lille

- août 1834 : expulsé vers la Savoie, via Lyon (refuse d'aller au Piémont)

- septembre 1834 : arrivée à Barcelone

26. Situation voisine dans le Morbihan : cas Perez (Arch. municipales de Vannes, 2 I 147).

27. Arch. dép. des Côtes-d'Armor, 4 M 205.

28. Arch. dép. des Côtes-d'Armor, 4 M 206.

29. Arch. dép. d'Ille-et-Vilaine, 4 M 352.

30. Arch. dép. du Morbihan, 4 M 389; Arch. municipales de Vannes, 2 I 147.

31. Arch. dép. des Côtes-d'Armor, 4 M 204.

32. Arch. dép. d'Ille-et-Vilaine, 4 M 352. 
- novembre 1834 : expulsé de Catalogne

- décembre 1834 : arrivée à Bayonne, reçoit du consul un passeport pour Pampelune pour servir le général Mina.

- mai 1835 : arrêté à Montpellier

- juin 1835 : conduit à Calais

-5 octobre 1835 : expulsé pour l'Angleterre

- 11 janvier 1836 : arrêté à Lons-le-Saulnier

- 6 janvier 1837 : expulsé par Calais

- mars 1837 : arrêté à Montpellier

- 14 avril 1837 : expulsé pour l'Angleterre

- 20 juillet 1837 : signalé devant partir pour Anvers et revenir en France pour assassiner Louis-Philippe

On comprend alors évidemment mieux que le modénan Antoine Ferrati, ancien maréchal-des-logis de gendarmerie (admis à Rodez, puis à Moulins, avant d'être affecté dans les Côtes-du-Nord) ait eu besoin du témoignage du $\mathrm{D}^{\mathrm{r}}$ Joseph Garavini, en 1837, pour ne pas être confondu ${ }^{33}$.

Le cas Ferranti prouve, en tout cas, les limites du système de surveillance. Au temps des dépôts, et à diverses reprises, plusieurs réfugiés avaient échappé à l'Administration. Mais, ensuite, le contrôle n'est pas toujours étanche. Par exemple, le romain Bertola s'évanouit des Côtes-duNord, alors qu'il devait être expulsé par Boulogne, de même, deux Espagnols, perdus par le préfet de la Lozère, ont quitté Mende sans prévenir et sont signalés au plan national. En 1837, Léon Babski - pourtant autorisé à prendre résidence à Rennes - est perdu par le préfet de SeineInférieure et ne sera jamais retrouvé ${ }^{34}$. Que dire, alors, du colonel Castillo de Velasco, assigné à Quintin avec sa femme et ses deux enfants et invité à quitter le département (malgré le soutien du maire et de "plusieurs personnes honorables " ayant pétitionné pour lui), qui est arrêté dans le Morbihan après avoir abandonné Périgueux en septembre $1844^{35}$ ? Encore faut-il signaler que la Bretagne, tardivement dotée d'un système ferré, n'est pas l'espace des plus nombreuses "évasions".

Enfin, sur le plan comportemental, l'Administration développe un modèle de rectitude axée autour du double impératif de modération et de dignité, le réfugié devant subir l'exil dans le calme et le silence. Ce modèle ne souffre guère l'exception puisqu'une fête patriotique organisée par les réfugiés portugais de Fougères en septembre 1829 provoque des colères locales (mais la sympathie des libéraux, dont fait partie l'officier de gendarmerie chargé du rapport) ${ }^{36}$. Et les critères sont sévères. Sur les 47 Polonais venant de Besançon pour les Côtes-du-Nord, en 1833, seuls 16 sont signalés pour "bonne conduite ", contre 4 "décriés pour dettes " et

33. Arch. dép. des Côtes-d'Armor, 4 M 204.

34. Arch. dép. d'Ille-et-Vilaine, 4 M 427.

35. Arch. dép. d'Ille-et-Vilaine, 4 M 31; Arch. dép. des Côtes-d'Armor, 4 M 204-206.

36. Arch. dép. d'Ille-et-Vilaine, 4 M 260 et 357 
5 " inquiets ", " dangereux " ou " très dangereux ${ }^{37}$ ". Une bagarre provoquée par 4 Polonais dans une auberge de Moncontour, en août 1833, entraîne leur condamnation en correctionnelle à 6-15 jours de prison et l'expulsion ultérieure - le 10 juin 1834 - de deux d'entre eux (Jacques Kawalski et Pierre Stankiewicz, derechef entraînés dans une affaire de corruption) en accord avec le décret rendu le 2 avril 1834 sur les expulsions ${ }^{38}$. En mars 1835 , Michel Pietrazewki subit le même sort pour vol et insultes à Perros, à l'instar du capitaine Ignace Podezaski qui, depuis son arrivée en 1833, traîne des dettes, se bagarre souvent à Plancoët et revient implorer grâce à SaintMalo (en 1843), pour revoir ses enfants, après son expulsion d'octobre 1841 vers l'Angleterre ${ }^{39}$. On pourrait aussi signaler le cas de l'étudiant en médecine, Denis Smotriez, qui tente de se suicider après avoir été éconduit par une jeune Rennaise et se retrouve affecté, d'office, à Granville ${ }^{40}$.

Cependant, et une fois encore, la sévérité administrative n'est pas univoque. Le lieutenant Charles Merbeller, signalé à Besançon comme ayant une "bonne conduite ", figure parmi les protagonistes de la bagarre de Moncontour, mais est autorisé à rester en France car il put payer dettes et dégâts provoqués; employé chez un négociant de Saint-Brieuc, mais licencié pour immoralité, il passa ensuite à Jersey, y tint un débit de boisson avec sa maîtresse pour revenir à Saint-Servan (1836) et y être souvent arrêté pour ivresse (avec violence contre les forces de l'ordre, ce qui lui vaut 15 jours de prison) après avoir travaillé deux mois au port; il repart en Angleterre, en novembre 1837, où il est soupçonné d'organiser des excursions politiques en Écosse et en Belgique (en tout cas il se marie avec une belge); mais on le retrouve plus tard dans le Morbihan (après avoir résidé dans le Finistère), en juin 1846, autorisé à prendre les eaux de Vichy avant de se signaler, une dernière fois, en novembre 1851, pour des opinions démocratiques " dangereuses ${ }^{41}$ ". L'inconstance administrative se repère aussi concernant Mathias Calbo, qui bénéficie de l'appui épiscopal briochin pour obtenir une autorisation ministérielle de résidence dans les Côtes-du-Nord, en 1847, affirme vouloir rentrer en Espagne par Bayonne, mais que l'on retrouve marchand de vins et chocolat, expulsé vers l'Angleterre par Granville en $1856^{42}$. Néanmoins, l'idéologie administrative est volontiers intégrée au registre d'action collective des réfugiés qui établissent leur propre contrôle social, comme en témoignent des documents pour se désolidariser des déviants, tel l'aveu, en décembre 1831, du colo-

37. Arch. dép. des Côtes-d'Armor, 4 M 199.

38. Arch. dép. des Côtes-d'Armor, 4 M 198-199; Arch. dép. d'llle-et-Vilaine, 4 M 353, 355-356.

39. Arch. dép. des Côtes-d'Armor, 4 M 202; Arch. dép. d'Ille-et-Vilaine, 4 M 355. Sur avis favorable du ministre de l'Intérieur, il est autorisé à rester en Ille-et-Vilaine.

40. Arch. dép. d'Ille-et-Vilaine, 4 M 354.

41. Arch. dép. des Côtes-d'Armor, 4 M 199, 202; Arch. dép. d'Ille-et-Vilaine, 4 M 355, 4 M 428; Arch. dép. du Morbihan, 4 M 389-390. Gouy, Jean-Claude, "Les réfugiés polonais au Puy après l'insurrection de 1830 ", Cahiers d'Histoire, 3, 1979, p. 83-87, signale les mêmes craintes infondées, sauf exception.

42. Arch. dép. des Côtes-d'Armor, 4 M 206; Arch. dép. du Morbihan, 4 M 390. 
nel Pinto Feio (du dépôt de Rennes) admettant qu'il " existe parmi nous des individus [...] qui ne se conduisent pas très bien " ou le refus du colonel Da Silva d'accueillir, au dépôt de Fougères, Francisco-Joze de Asis signalé successivement à Laval, Nantes et Paris pour jeu et escroquerie, un peu à l'instar du sous-lieutenant de Mello qui meurt à l'hôpital Saint-Yves de Rennes (en juin 1833) après une vie qualifiée de " dissolue ${ }^{43}$ ".

Au bout du compte et parce qu'elle est mal renseignée sur une majorité d'individus ne posant guère de difficultés, l'Administration se fie au principe de délégation avec une telle bonhomie, parfois, que le Ministre doive rappeler à l'ordre les préfets pour qu'ils exécutent les relevés demandés.

\section{L'exil : vivre ou en mourir}

Bien souvent dominée par des contraintes matérielles qui émaillent les dossiers des réfugiés - surtout, et c'est chose commune, lorsque les subsides sont versés avec retard - la vie d'exil conjugue souvent oisiveté et endettement (que règlent, en partie, les retenues sur subsides). Nous n'y insisterons pas. Reste donc l'épineux problème de l'intégration éventuelle. Quatre grands cas de figures se présentent.

D'abord, et selon les vœux même de l'État, un certain nombre de réfugiés, dont la majorité a 25-35 ans en arrivant en France, obtient d'entamer ou de poursuivre des études. Mais celles-ci sont sévèrement contrôlées, et rien n'est gagné puisque si Alexandre Szuskiewicz est qualifié d'élève " distingué de l'école préparatoire de médecine de Rennes »- mais on ignore le reste de sa carrière, - Adolphe Filipowicz, arrivé en France en 1832, reçu second lauréat du premier concours établi par l'École en 1841, diplômé de médecine en 1844 et qui s'installe à Médréac (Ille-et-Vilaine) traîne une difficile carrière jusque 1857 et des problèmes d'intégration ${ }^{44}$. Quant à l'étudiant Antoine Lazowski, souhaitant aller à Paris poursuivre sa formation en pharmacie, il est affecté à Poitiers (pour passer finalement en Belgique); la même destination est requise pour les étudiants en droit Pierre Ucellini, Jean-Baptiste Prampolini, Jules Gabrielli, Antoine Aguzolli, François Montanari dont on ne connaît pas le sort ultérieur ${ }^{45}$. En revanche, quelques réfugiés parviennent à exercer leur activité passée, comme Louis della Casa (stucateur), Lucien Rossi (sculpteur), les médecins Joseph Garavini (Lamballe) et François Montanari (Lorient) ${ }^{46}$. On pourrait y rattacher Luigi Odorici (1806-1882), ancien professeur de littérature à Modène et qui, à

43. Arch. dép. d'Ille-et-Vilaine, 4 M 362 et 364.

44. Bibliothèque municipale de Rennes, Ms. 807, Registre des séances et délibérations de la Société libre d'enseignement de médecine..., séance du 2 août 1841 (citée par TRUMIER, Jean-François, Une assemblée savante de province : la Société de médecine de Rennes (1800-1860), maîtrise, Rennes 2, dir. P. Harismendy, 1998, p. 58-59); Arch. dép. d'Ille-et-Vilaine, 4 M 427.

45. Arch. dép. d'Ille-et-Vilaine, 4 M 356; Arch. dép. des Côtes-d'Armor, 4 M 202; Arch. dép. du Morbihan, 4 M 389.

46. Arch. dép. des Côtes-d'Armor, 4 M 204; Arch. dép. du Morbihan, 1 Z 78; 4 M 389. 
force de suppliques, d'envois dédicacés de ses œuvres aux préfets et ministres, finit par devenir conservateur de la bibliothèque de Dinan en 1842, collectionneur, minéralogiste et érudit local ${ }^{47}$; ou encore Constantin Kozerawski, dont le fils Alexandre entre au service du Conseil général d'Illeet-Vilaine et dont le petit-fils, Charles (né en 1862), fonde, en 1911, la Société des Amis de Vannes, toujours active ${ }^{48}$.

La seconde catégorie d'individus - la plus intéressante au plan social est formée exclusivement de Polonais (sauf Gaëtan Tempellini employé chez un géomètre) qui s'attachent aux corps techniques et, plus précisément, entrent au service des Ponts-et-chaussées. Aidée par l’État, la diaspora avait mis en place diverses structures d'intégration, notamment la Société polonaise des amis du progrès dans l'industrie ou la Société polytechnique polonaise qui semble avoir aidé Antoine Patek, Alexandre Marchoki, François Obuchowiez et Diomède Tuczkiewicz à entrer dans des entreprises amiénoises, en 1834, sur recommandation du Prince de Joinville ${ }^{49}$. En l'état actuel, il est difficile de dresser une liste complète de tous ces employés - surtout pour les catégories inférieures, du reste toujours très mal renseignées, - mais on possède au moins des attestations de services pour un certain nombre de personnes dans les Côtes-du-Nord et l'Ille-et-Vilaine (voir liste en annexes), qui illustrent le débordement de l'activité technicienne au-delà des seules fonctions (assez prestigieuses) de conducteur ${ }^{50}$. L'attestation de ces volumes importants ne résout pas la question de fond qui est de savoir par quels canaux tous ces hommes, issus d'armes différentes (génie et artillerie ne prévalant pas comme on aurait pu s'y attendre), ont pu accéder à ces fonctions. Fonctions d'ailleurs très différentes, car la surveillance de travaux vicinaux ne requiert pas de compétences particulières mais plutôt ponctualité et autorité, tandis que plusieurs conducteurs notés comme " ayant peu de sciences " par les ingénieurs présentent la caractéristique majeure de "savoir dessiner " ou d'être de " constitution robuste ». De surcroît, si Odelski, Roguski, Rozanski et Roginski se connaissaient bien pour avoir témoigné les uns pour les autres - comme Stryjenski, Piotrowski, Erdmann, Musniecki, - ou avoir cosigné une demande d'admission dans la Légion étrangère (refusée comme

47. Monier, M.-E, Dinan, 1000 ans d'histoire, Mayenne, Floch, 1968, p. 557; on lui doit, notamment, les traductions de Silvio Pellico, Devoirs de l'homme, discours à un jeune homme, Saint-Brieuc, 1834, Ir-136 p.; de l'abbé Giuseppe Taverna, Historiettes morales de Taverna, Dinan, 1836, 165 p.; la composition de Claremont-Weybridge, 1848-1866, notes et souvenirs extraits de mon journal, Dinan, 1871, VII-167 p.; Du Guesclin, documents inédits et peu connus relatifs à la découverte du cour de Du Guesclin dans l'église des Jacobins de Dinan et à sa translation dans celle de Saint-Sauveur de la même ville, précédés d'une notice biographique, Dinan, 1850, IV-63 p.; Recherches sur Dinan et ses environs, Dinan, 1857, 636 p.

48. Bulletin des Amis de Vannes, 1, 1975, p. 12; 15, 1990, p. 19.

49. Arch. municipales de Vannes, 2 I 147.

50. Arch. dép. des Côtes-d'Armor, 4 M 200-204; 203; 1 S 3 (12) IV ; Arch. dép. d'Ille-etVilaine, 4 M 356; Arch. dép. du Morbihan, 4 M 387; Arch. nat. F14 2474, 2645, 2676 (dossiers de Chamski, Piotrowski, Stryenski aimablement communiqués par Jean Le Bihan). 
à tous les officiers car on n'avait besoin que de soldats), le mécanisme de solidarité demeure inconnu.

Autre inconnue, mais sur laquelle le fichier nominatif de Christian Priet sur la population rennaise au XIX ${ }^{\mathrm{e}}$ siècle fournit de très précieux éclairages, la question des unions. En se limitant là encore aux seuls Polonais, prévaut une certaine diversité, même si l'on est finalement surpris par l'importance relative de volumes insoupçonnables à travers les dossiers administratifs.

\begin{tabular}{|c|c|c|c|c|c|}
\hline $\begin{array}{l}\text { Nom et année } \\
\text { de naissance }\end{array}$ & Activité en exil & $\begin{array}{c}\text { Épouse et } \\
\text { date de mariage }\end{array}$ & $\begin{array}{l}\text { Activité de } \\
\text { l'épouse }\end{array}$ & $\begin{array}{l}\text { Activité du } \\
\text { beau-père }\end{array}$ & $\begin{array}{l}\text { Nombre } \\
\text { d'enfants } \\
\text { connus }\end{array}$ \\
\hline $\begin{array}{c}\text { Erdmann, } \\
\text { Julien (1802) }\end{array}$ & $\begin{array}{c}\text { Employé des } \\
\text { Ponts-et- } \\
\text { chaussées }\end{array}$ & $\begin{array}{c}\text { Boudry, Rosalie } \\
\text { (juin 1843) }\end{array}$ & Charcutière & $\begin{array}{l}\text { Receveur } \\
\text { de l'octroi }\end{array}$ & \\
\hline $\begin{array}{l}\text { Falkowsky, } \\
\text { Jules (1816) }\end{array}$ & Ingénieur civil & $\begin{array}{c}\text { Bodin, Elena } \\
\text { (décembre 1858) }\end{array}$ & Sans & $\begin{array}{c}\text { Chef de } \\
\text { la musique } \\
\text { militaire }\end{array}$ & \\
\hline \begin{tabular}{c|} 
Filanowicz, \\
Faustin (1816)
\end{tabular} & $\begin{array}{c}\text { Employé des } \\
\text { chemins } \\
\text { vicinaux }\end{array}$ & $\begin{array}{c}\text { Vit maritalement } \\
\text { avec Georgina } \\
\text { Angelo }\end{array}$ & Sans & Inconnu & $\begin{array}{c}\text { Une fille } \\
\text { naturelle recon- } \\
\text { nue en } 1839 \\
\end{array}$ \\
\hline \begin{tabular}{c|} 
Hofmann, \\
Frédéric (1817)
\end{tabular} & $\begin{array}{l}\text { Sellier, puis } \\
\text { carrossier }\end{array}$ & $\begin{array}{c}\text { Macé, Françoise } \\
\text { (octobre 1848) }\end{array}$ & Sans & Cordonnier & Un \\
\hline $\begin{array}{c}\text { Jaworsky, } \\
\text { Jean (1797) }\end{array}$ & $\begin{array}{l}\text { Cafetier, puis } \\
\text { concierge }\end{array}$ & $\begin{array}{c}\text { Maguet, Mathilde } \\
\text { (juillet 1847) }\end{array}$ & $\begin{array}{c}\text { Marchande de } \\
\text { café }\end{array}$ & Soldat & Trois \\
\hline \begin{tabular}{c|} 
Jezwski, \\
Erasme (1814)
\end{tabular} & Sans & $\begin{array}{c}\text { Chevrier, } \\
\text { Julienne (?) }\end{array}$ & Sans & Peintre-vitrier & Un \\
\hline $\begin{array}{c}\text { Kutscki, } \\
\text { André (1802) }\end{array}$ & Cordonnier & $\begin{array}{c}\text { Lemel, } \\
\text { Antoinette (?) }\end{array}$ & Tricoteuse & Inconnu & \\
\hline $\begin{array}{c}\text { Maxa, } \\
\text { Mathias (1806) }\end{array}$ & Piqueur & $\begin{array}{c}\text { Jamain, } \\
\text { Anatolie (1841) }\end{array}$ & Sans & Inconnu & Deux \\
\hline $\begin{array}{l}\text { Piotrowsky, } \\
\text { Denis (1805) }\end{array}$ & $\begin{array}{l}\text { Conducteur } \\
\text { des Ponts-et- } \\
\text { chaussées }\end{array}$ & $\begin{array}{c}\text { Fenwick, } \\
\text { Isabella } \\
\text { (juillet 1843) }\end{array}$ & Sans & $\begin{array}{l}\text { Rentier } \\
\text { (anglais) }\end{array}$ & Cinq \\
\hline $\begin{array}{l}\text { Ramotowski, } \\
\text { Joseph (?) }\end{array}$ & Inconnue & $\begin{array}{c}\text { Guesdon } \\
\text { de Beauchesne, } \\
\text { Constance (?) }\end{array}$ & Sans & Inconnu & Deux \\
\hline $\begin{array}{c}\text { Wolski, } \\
\text { Joseph (1802) }\end{array}$ & Sans & $\begin{array}{c}\text { Chalais, Marie } \\
\text { (septembre } \\
1841 \text { ) }\end{array}$ & Sans & Meunier & Deux \\
\hline $\begin{array}{c}\text { Zielinski, } \\
\text { Sabin (1813) }\end{array}$ & $\begin{array}{l}\text { Employé de } \\
\text { préfecture }\end{array}$ & $\begin{array}{c}\text { Nourry, } \\
\text { Louise (?) }\end{array}$ & Sans & Inconnu & Deux \\
\hline
\end{tabular}

Subsiste, enfin, le groupe peu nombreux de ceux qui restent dans les trois départements, faute d'autres perspectives (amnistie, emploi, mariage avantageux, émigration...) et, bien souvent, traînent blessures physiques ou morales. À cette catégorie se rattachent, notamment, les cas des souslieutenants Lucas Jasniewicz (assigné à Lannion depuis 1835 et qui y meurt en 1854) et François Midwock (à Paimpol de mai 1833 à sa mort en novembre 1856), du capitaine Antoine Konopacki incapable d'apprendre le français et adressant ses requêtes en polonais ou en latin (pour mourir à 
Rennes en 1859 après avoir été à Quintin et à Vannes) ${ }^{51}$. Plus pathétique est l'issue du lieutenant modénan Hippolyte Forti, admis à Rennes en 1836 - déjà en très mauvaise santé à la suite de blessures, - qui contracte des dettes pour se soigner à Mordelles avant d'être admis à l'hôpital Saint-Yves de Rennes, rejoint Redon pour échapper à ses créanciers (avec l'accord de l'Administration) et s'y suicide en mai $1840^{52}$. Quant au doyen des subventionnés des trois départements, il semble que ce fut Telmo Goncalves Doval, ancien commissaire des guerres carliste, arrivé en 1839 à Dinan (très surveillé en raison de ses idées ultra) et qui déclina dans un état de misère et d'impotence mystique effroyables (malgré l'aide de la municipalité et de quelques personnes charitables) pour mourir en décembre $1877^{53}$.

Dans ces réalités, souvent insaisissables, quelques observations peuvent être faites. D'abord, si le phénomène d'exil politique est nettement borné dans le temps mais s'accompagne d'intégrations locales jusqu'alors insoupçonnées, la mort dans l'indigence reste la norme, même pour beaucoup d'employés (qui le sont rarement en continu et se retrouvent presque toujours exploités). D'autre part, le retour à des situations professionnelles compatibles avec les niveaux socio-professionnels d'origine sont rarissimes et prouvent que l'exil constitue un risque et une flétrissure. À cet égard, les administrations municipales et préfectorales ont toujours défendu le maintien des subsides contre les coupes sombres réclamées par Paris. Enfin, l'on reste très mal informé des réseaux de soutien (avec les populations d'accueil) ou des solidarités (entre réfugiés).

Par conséquent, et pour en savoir davantage, d'autres méthodes ou sources devraient être mobilisées. Ainsi, l'analyse micro-historique concernant quelques individus (en évitant de se contenter des apax) ou certaines communes (comme Dinan, Fougères ou Hédé) éclaireraient sans doute la vie quotidienne et réelle de tels milieux. De même qu'une enquête nationale - voire internationale, dans l'idéal - et la constitution d'un fichier étendu aux descendants modifieraient, sans doute, l'aperçu trop négatif quant à l'intégration. Quant à la littérature de l'exil ou, plus largement, de mémoires d'exilés (au besoin retournés dans leur patrie d'origine) elle renverrait, probablement, une autre lueur sur ces vies incertaines et à bien des égards tragiques.

51. Arch. dép. des Côtes-d'Armor, 4 M 202.

52. Arch. dép. d'llle-et-Vilaine, 4 M $351 ; 3$ M 389.

53. Arch. dép. des Côtes-d'Armor, 4 M 205-206. 


\section{Annexe 1 - Personnel polonais des Travaux publics dans les Côtes-du-Nord}

\begin{tabular}{|c|c|c|c|}
\hline Nom & $\begin{array}{l}\text { Origine } \\
\text { professionnelle }\end{array}$ & $\begin{array}{l}\text { Lieu de dépôt ou } \\
\text { d'origine dans l'exil }\end{array}$ & Affectations \\
\hline Bagienski Stanislas & $\begin{array}{l}\text { Sorti du rang } \\
\text { Capitaine d'artillerie }\end{array}$ & Besançon (1832) & $\begin{array}{l}\text { Employé aux Ponts-et- } \\
\text { chaussées chez son cousin, } \\
\text { conducteur, Zabkovski } \\
\text { (Valence, 1844) }\end{array}$ \\
\hline Besiecki Valentin & $\begin{array}{l}\text { Capitaine } \\
\text { d'infanterie }\end{array}$ & & $\begin{array}{l}\text { Cantonnier-chef dans } \\
\text { les Côtes-du-Nord }\end{array}$ \\
\hline Horain Michel (de) & $\begin{array}{l}\text { Études à l'université } \\
\text { de Vilnius } \\
\text { Major du génie }\end{array}$ & $\begin{array}{l}\text { Besançon (1832) } \\
\text { St-Brieuc (1833-1835) }\end{array}$ & $\begin{array}{l}\text { Conducteur de } 3^{\mathrm{e}} \text { classe } \\
\text { (Tours; 1835) }\end{array}$ \\
\hline Klodowski Theodor & & & $\begin{array}{l}\text { Piqueur dans } \\
\text { les Côtes-du-Nord (1837) }\end{array}$ \\
\hline $\begin{array}{l}\text { Kossack Charles } \\
(1807-1833) \\
\text { se suicide }\end{array}$ & $\begin{array}{l}\text { Sous-lieutenant } \\
\text { de cavalerie }\end{array}$ & Vient du Morbihan & $\begin{array}{l}\text { Devait être employé aux } \\
\text { travaux du canal } \\
\text { de Nantes à Brest }\end{array}$ \\
\hline Odelski François & Capitaine d'artillerie & Besançon (1832) & $\begin{array}{l}\text { Cantonnier-chef dans } \\
\text { les Côtes-du-Nord }\end{array}$ \\
\hline $\begin{array}{l}\text { Roginski Joseph } \\
(1799-1858)\end{array}$ & $\begin{array}{l}\text { Lieutenant } \\
\text { d'infanterie }\end{array}$ & Besançon (1832) & $\begin{array}{l}\text { Surveillant des chemins } \\
\text { vicinaux (1844-1853) }\end{array}$ \\
\hline $\begin{array}{l}\text { Roguski Mathias } \\
\text { (né en 1802) }\end{array}$ & $\begin{array}{l}\text { Sous-lieutenant de } \\
\text { chasseurs à cheval }\end{array}$ & Besançon (1832) & $\begin{array}{l}\text { Piqueur aux Ponts-et- } \\
\text { chaussées (1837-1841) } \\
\text { Employé auxiliaire de } 2^{\text {e }} \text { classe } \\
\text { (1841-1852) } \\
\text { Employé auxiliaire de } 1^{\text {re }} \text { classe } \\
\text { (1854) } \\
\text { Encore actif en } 1869\end{array}$ \\
\hline $\begin{array}{l}\text { Rozanski Felix } \\
(1803-1867)\end{array}$ & $\begin{array}{l}\text { Sous-lieutenant de } \\
\text { lanciers }\end{array}$ & Besançon (1832) & $\begin{array}{l}\text { Piqueur aux Ponts-et- } \\
\text { chaussées - Conducteur }\end{array}$ \\
\hline $\begin{array}{l}\text { Siedlecki Paul- } \\
\text { Alexandre } \\
(1810-1852)\end{array}$ & $\begin{array}{l}\text { Lieutenant } \\
\text { d'artillerie }\end{array}$ & $\begin{array}{l}\text { Certificat médical } \\
\text { du } D^{r} \text { Hamel } \\
\text { (Lannion, juillet 1835) } \\
\text { pour schizophrénie }\end{array}$ & $\begin{array}{l}\text { Employé des chemins } \\
\text { vicinaux, agent-voyer } \\
\text { de Corlay (1837-1852) }\end{array}$ \\
\hline $\begin{array}{l}\text { Szabuniewicz } \\
\text { Stanislas (1809-1860) } \\
\text { se suicide }\end{array}$ & $\begin{array}{l}\text { Capitaine } \\
\text { d'infanterie }\end{array}$ & & $\begin{array}{l}\text { Employé aux chemins de fer } \\
\text { à Strasbourg } \\
\text { Conducteur auxiliaire dans les } \\
\text { Côtes-du-Nord (1849) }\end{array}$ \\
\hline $\begin{array}{l}\text { Wisniewski } \\
\text { Maximilien } \\
(1809-1872)\end{array}$ & $\begin{array}{l}\text { Lieutenant } \\
\text { d'artillerie }\end{array}$ & $\begin{array}{l}\text { Actif au chemin } \\
\text { de fer Paris- } \\
\text { Strasbourg }\end{array}$ & $\begin{array}{l}\text { Conducteur à Guingamp } \\
(1844-1861) \\
\text { Nommé en Seine-et-Marne }\end{array}$ \\
\hline Zombrowski Valentin & Sous-lieutenant & $\begin{array}{l}\text { Vient de Blois et } \\
\text { Paris - Arrivé en } 1835 \\
\text { dans les Côtes- } \\
\text { du-Nord }\end{array}$ & $\begin{array}{l}\text { Conducteur auxiliaire de } \\
3^{\mathrm{e}} \text { classe (1837) } \\
\text { Conducteur auxiliaire de } \\
2^{\mathrm{e}} \text { classe (1839) } \\
\text { Passe en Isère (1844) } \\
\text { Retraite (?) (1864) }\end{array}$ \\
\hline
\end{tabular}


Annexe 2 - Personnel polonais des Travaux publics en Ille-et-Vilaine

\begin{tabular}{|c|c|c|c|}
\hline Nom & $\begin{array}{l}\text { Origine } \\
\text { professionnelle }\end{array}$ & $\begin{array}{l}\text { Lieu de dépôt ou } \\
\text { d'origine dans l'exil }\end{array}$ & Affectations \\
\hline $\begin{array}{l}\text { Chamski Thadée } \\
\text { (1805-1881) }\end{array}$ & $\begin{array}{l}\text { Lieutenant sorti } \\
\text { du rang }\end{array}$ & Rennes & $\begin{array}{l}\text { Piqueur } \\
\text { Conducteur auxiliaire } \\
\text { (1847-1859) } \\
\text { Conducteur embrigadé } \\
\text { (1859-1878) } \\
\text { Retraité comme embrigadé } \\
\text { de } 2^{\mathrm{e}} \text { classe (1878) }\end{array}$ \\
\hline $\begin{array}{l}\text { Erdmann Julien } \\
(1802-)\end{array}$ & $\begin{array}{l}\text { Juge de paix } \\
\text { Sous-lieutenant de } \\
\text { partisans }\end{array}$ & $\begin{array}{l}\text { Présent en Ille-et- } \\
\text { Vilaine (1844) }\end{array}$ & $\begin{array}{l}\text { Employé auxiliaire au } \\
\text { bureau de l'ingénieur-en-chef } \\
\text { Conducteur des Ponts-et- } \\
\text { chaussées }\end{array}$ \\
\hline $\begin{array}{l}\text { Filanowiez Faustin } \\
(1816-)\end{array}$ & Lieutenant d'artillerie & \begin{tabular}{|l|} 
Réside en Ille-et- \\
Vilaine puis Morbihan
\end{tabular} & $\begin{array}{l}\text { Part pour Paris comme } \\
\text { conducteur auxiliaire (1845) } \\
\text { Retraité (1882) }\end{array}$ \\
\hline $\begin{array}{l}\text { Katuzynski Jacques } \\
\text { (1804-) }\end{array}$ & $\begin{array}{l}\text { Propriétaire } \\
\text { Sous-lieutenant de } \\
\text { volontaires }\end{array}$ & Vitré (1834-1845) & $\begin{array}{l}\text { Employé comme chef d'atelier } \\
\text { des ponts-et-chaussées au } \\
\text { dépôt de Vitré }\end{array}$ \\
\hline Krackiewiez Martin & Capitaine du génie & Saint-Servan & $\begin{array}{l}\text { Conducteur auxiliaire à la } \\
\text { construction des bassins à flot } \\
\text { de Saint-Servan }\end{array}$ \\
\hline $\begin{array}{l}\text { Kreczkowski } \\
\text { Alexandre (1804-1849) }\end{array}$ & Sous-lieutenant & Saint-Servan & $\begin{array}{l}\text { Employé à la construction } \\
\text { des bassins à flot de } \\
\text { Saint-Servan (1841-1844) }\end{array}$ \\
\hline $\begin{array}{l}\text { Maxa Mathias } \\
(1806-1871)\end{array}$ & $\begin{array}{l}\text { Sous-lieutenant de } \\
\text { Cavalerie }\end{array}$ & $\begin{array}{l}\text { Haute-Vienne } \\
\text { Attesté à Fougères } \\
(1856)\end{array}$ & $\begin{array}{l}\text { Mécanicien à Louviers } \\
\text { Piqueur aux chemins } \\
\text { de fer du Nord }\end{array}$ \\
\hline $\begin{array}{l}\text { Musniecki de Janjsol } \\
\text { Napoléon } \\
(1814-)\end{array}$ & $\begin{array}{l}\text { Sous-lieutenant } \\
\text { d'infanterie } \\
\text { Etudiant en droit }\end{array}$ & Rennes & $\begin{array}{l}\text { Piqueur des ponts-et- } \\
\text { chaussées (1838-1843) } \\
\text { Employé au bureau } \\
\text { de l'ingénieur ordinaire } \\
\text { des ponts-et-chaussées } \\
\text { de Rennes (1843-1856) }\end{array}$ \\
\hline $\begin{array}{l}\text { Piotrowski Denis } \\
\text { (1805-) }\end{array}$ & & & $\begin{array}{l}\text { Conducteur auxiliaire } \\
(1843-1852) \\
\text { Conducteur embrigadé } \\
(1852-1862) \\
\text { Démissionnaire (1862) }\end{array}$ \\
\hline $\begin{array}{l}\text { Stryjienski Paul } \\
(1814-)\end{array}$ & $\begin{array}{l}\text { Sous-lieutenant } \\
\text { d'infanterie }\end{array}$ & Rennes & $\begin{array}{l}\text { Employé auxiliaire (1838-1840) } \\
\text { Piqueur (1840) } \\
\text { Conducteur auxiliaire } \\
\text { (1842-1852) } \\
\text { Conducteur embrigadé } \\
\text { (1852-1856) } \\
\text { Contrôleur du chemin de fer } \\
\text { Rennes-Saint-Malo (1856-1857) } \\
\text { En congé illimité (1857) } \\
\text { Retraité (1878) }\end{array}$ \\
\hline $\begin{array}{l}\text { Witkowski, } \\
\text { Maximilien (1814-) }\end{array}$ & $\begin{array}{l}\text { Employé des ponts- } \\
\text { et-chaussées } \\
\text { Sous-lieutenant }\end{array}$ & $\begin{array}{l}\text { Besançon } \\
\text { Rennes }\end{array}$ & $\begin{array}{l}\text { Employé agent-voyer } \\
\text { (1838-1842) } \\
\text { Piqueur au canal d'Ille-et- } \\
\text { Rance (1843-1845) }\end{array}$ \\
\hline
\end{tabular}


Les réfugiés politiques en Bretagne (1830-1848)

\begin{tabular}{|l|l|l|l|}
\hline $\begin{array}{l}\text { Wozcickowski Louis } \\
\text { (se suicide en 1840) }\end{array}$ & & $\begin{array}{l}\text { Employé à la direction des } \\
\text { Ponts-et-chaussées d'Ille-et- } \\
\text { Vilaine comme conducteur } \\
\text { de 2e classe }\end{array}$ \\
\hline $\begin{array}{l}\text { Zcepanovski } \\
\text { Balthazar }\end{array}$ & Sous-lieutenant \\
\hline $\begin{array}{l}\text { Zielinski Sabin } \\
(1813-1892)\end{array}$ & $\begin{array}{l}\text { École polytechnique } \\
\text { de Varsovie } \\
\text { Sous-lieutenant } \\
\end{array}$ & & $\begin{array}{l}\text { Part à Dieppe pour le service } \\
\text { des Ponts-et-chaussées (1837) }\end{array}$ \\
& & $\begin{array}{l}\text { Employé au service vicinal } \\
\text { Agent-voyer cantonal de } \\
\text { Rennes, Saint-Malo, Vitré } \\
\text { (1839-1843) } \\
\text { Agent-voyer d'arrondissement } \\
\text { de Vitré puis Rennes } \\
(1843-1859) \\
\text { Ingénieur civil (1846) } \\
\text { Chef de bureau, puis chef de } \\
\text { division à la préfecture d'llle- } \\
\text { et-Vilaine (1859-1880) }\end{array}$ \\
\hline
\end{tabular}




\section{RESUME}

Les réfugiés politiques placés en Bretagne de la fin de la Restauration à la Seconde république appartinrent à quatre espaces nationaux : Espagne, Portugal, Italie et Pologne. A travers plusieurs sources, il ressort que des itinéraires individuels cohabitèrent avec des comportements collectifs. Cependant, et malgré la difficulté à brosser des conclusions, il semble qu'entre la politique étatique et des ajustements locaux, les réfugiés bénéficièrent d'une certaine latitude correspondant à leur désir de vivre en paix. En tout état de cause, les espoirs d'intégration furent restreints par les barrières linguistiques, des détresses psychologiques ou des incompatibilités professionnelles. Cette étude et ses prolongements possibles invitent à poursuivre la réflexion en dépit des problèmes méthodologiques rencontrés.

\section{ABSTRACT}

Political refugees in Britanny since the end of Restoration time to Second Republic belonged to four nations : Spain, Portugal, Italy and Poland. Through different sources it appears that singular itineraries were placed side by side with more massive behaviours. Though conclusions are difficult to stress, it seems that State policy and local managements let a rather important freedom to refugees, longing for individual peace. Meanwhile, hopes for integration were challenged by linguistic boundaries, psychological distresses and professionnal disabilities. This study case and its possible developments invites to further researches in spite of methodological difficulties. 